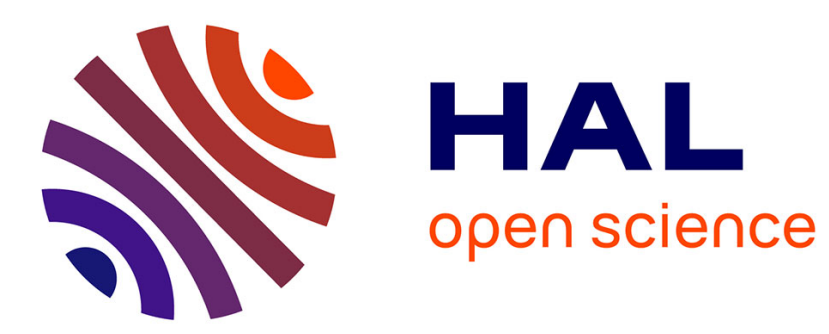

\title{
Dépérissement local du Pin d'Alep (Pinus halepensis) le long du littoral méditerranéen
}

\author{
Pierre-Marie Badot, Jean-Pierre Garrec
}

\section{To cite this version:}

Pierre-Marie Badot, Jean-Pierre Garrec. Dépérissement local du Pin d'Alep (Pinus halepensis) le long du littoral méditerranéen. Revue forestière française, 1993, 45 (2), pp.134-140. 10.4267/2042/26404 . hal-03444067

\section{HAL Id: hal-03444067 \\ https://hal.science/hal-03444067}

Submitted on 23 Nov 2021

HAL is a multi-disciplinary open access archive for the deposit and dissemination of scientific research documents, whether they are published or not. The documents may come from teaching and research institutions in France or abroad, or from public or private research centers.
L'archive ouverte pluridisciplinaire HAL, est destinée au dépôt et à la diffusion de documents scientifiques de niveau recherche, publiés ou non, émanant des établissements d'enseignement et de recherche français ou étrangers, des laboratoires publics ou privés. 


\title{
DÉPÉRISSEMENT LOCAL DU PIN D’ALEP (PINUS HALEPENSIS) LE LONG DU LITTORAL MÉDITERRANÉEN
}

\author{
P.-M. BADOT - J.-P. GARREC
}

Au cours des dernières années, un nombre considérable de travaux ont eu pour but de comprendre les nouveaux phénomènes de dèperissement qui affectent de nombreuses forêts dans le monde. Dans les grandes régions forestières d'Europe, ces dépérissements apparaissent aujourd'hui stabilisès. Ils n'en demeurent pas moins préoccupants (Bonneau et Landmann, 1988). S'ils n'ont plus la faveur des media, ils ont cependant permis de susciter une large prise de conscience collective. Les polluants atmosphériques, et plus particulièrement l'ozone et le dioxyde de soufre, ont souvent été mis en cause comme un des facteurs responsables des dégâts aux forêts (Garrec, 1991). La part prise par les èpisodes de sécheresses dans la genèse de ces phénomènes a également été clairement établie (Becker, 1991). A côtè de ces deux facteurs de dépérissement, d'autres agents sont également susceptibles localement de porter gravement atteinte à la végétation. En particulier, les effets phytotoxiques des embruns marins pollués sur la végétation littorale sont beaucoup moins connus.

Pourtant, dès les années 1950, les premières atteintes étaient signalées au voisinage de l'agglomération marseillaise (Devèze et Sigoillot, 1978), puis sur la côte tyrrhénienne en Italie (Lapucci et al., 1972). Les observations effectuées depuis lors indiquent que le mal s'est régulièrement aggravé : les dommages sont actuellement visibles sur de nombreux sites du littoral méditerranéen. Les côtes atlantiques seraient également touchées. Ce phénomène n'est pas propre aux rivages français. Des publications étrangères font état de problèmes graves affectant la végétation littorale en Italie (Gellini et al., 1983) et en Australie (Dowden et Lambert, 1979). Toutes les espèces sont touchèes y compris les halophiles et, au niveau des arbres, il semble que les essences à feuilles persistantes sont les plus défavorisées en raison de l'accumulation des polluants.

La végétation forestière située en bord de mer joue un très grand rôle tant sur le plan écologique qu'aux plans économique ou humain et les intérêts, qu'il y a à la protéger, sont évidents. À ce jour, seul un petit nombre de travaux a été consacré à l'étude des effets des embruns marins polluès sur la végètation et les informations disponibles sont fragmentaires. L'apparition des dépérissements serait consécutive à la présence dans les embruns de substances phytotoxiques. L'implication de detergents et de produits pétroliers dans ces dépérissements littoraux a été mise en évidence par Sigoillot et al. (1981). Nous avons entrepris d'étudier les mécanismes exacts de la phytotoxicité de ces substances. Une étape préliminaire consiste à caractériser avec précision les symptômes macroscopiques observés sur les arbres atteints. Le Pin d'Alep est l'une des essences les plus communes et les plus affectées du littoral méditerranéen. II constitue de ce fait un matériel de choix pour une telle étude. Cet article présente aussi quelques informations concernant la localisation et l'intensité du dépérissement de cette essence et fait le point des données actuelles relatives au mode d'action des embruns pollués. 


\section{LES PRINCIPAUX SYMPTÔMES DU DÉPÉRISSEMENT DU PIN D'ALEP SOUS L'EFFET DES EMBRUNS MARINS POLLUÉS}

La photographie 1a (ci-dessous) montre l'aspect d'un Pin d'Alep, planté dans le massif du Dramont (commune de Saint-Raphaël, Var), à proximité immédiate de la mer. Cet arbre est soumis à l'effet des embruns sur sa face directement exposée au vent de mer (à droite sur la photographie). Le port en drapeau, fréquent chez un arbre de bord de mer, indique le sens des vents dominants, qui sont ici orientes sud-est. Les branches et les rameaux qui reçoivent ces vents en provenance du large sont entièrement défoliés. Les aiguilles subsistent uniquement du côté terre où elles bénéficient d'une relative protection par le reste de l'arbre.

La photographie 1b (ci-dessous) fournit un exemple analogue ayant trait à des individus présentant un port buissonrant.

Photo 1

Pin d'Alep au cap du Dramont.

a: la face exposée au vent de mer est entièrement défoliée.

$\mathrm{b}$ : arbres à port buissonnant, des aiguilles subsistent sur la face qui bènéficie d'une protection relative vis-à-vis des embruns.

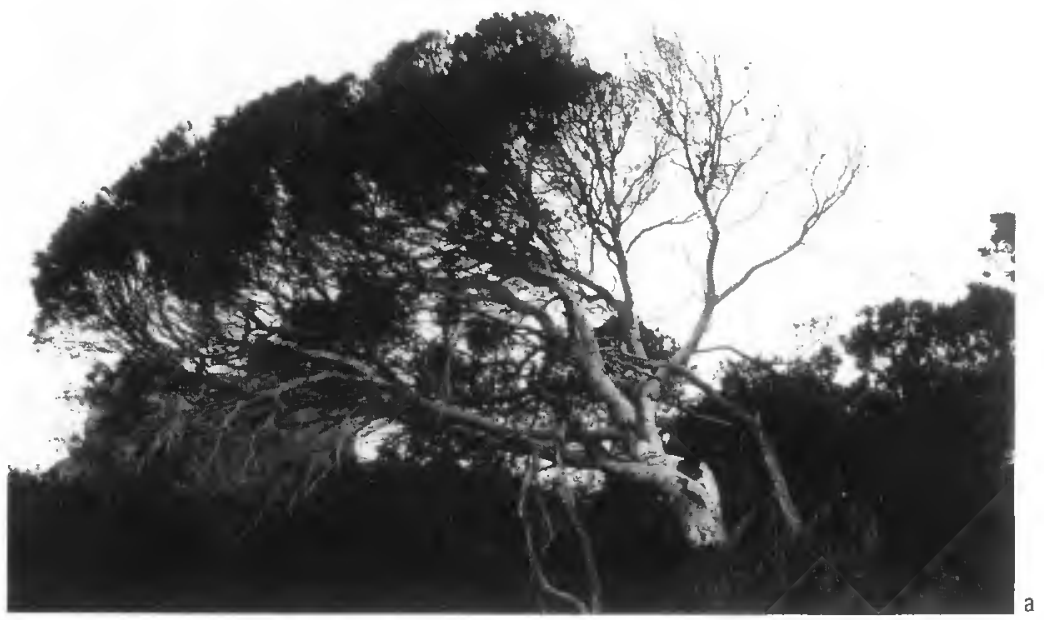

b

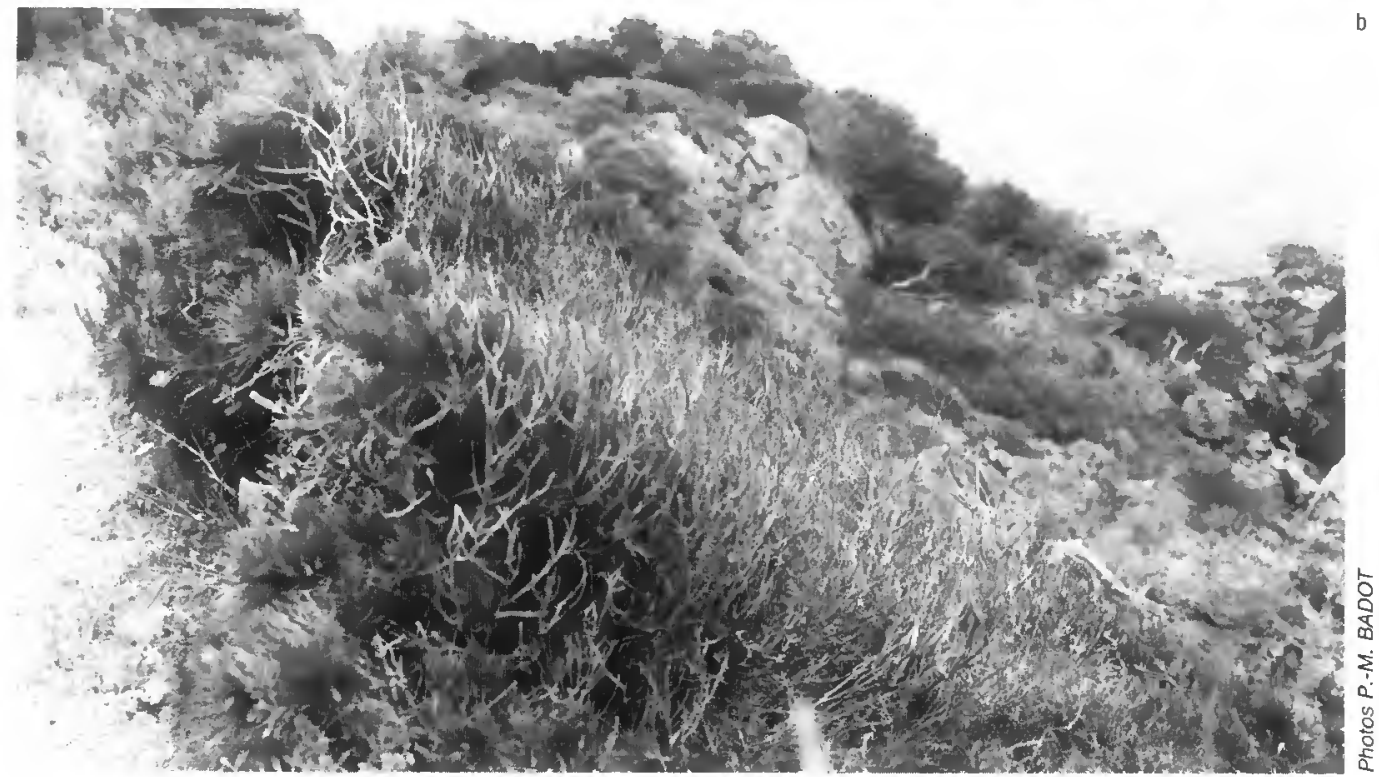


Trois zones peuvent être distinguées. La première correspond aux branches directement soumises au contact des embruns : la défoliation $y$ est totale. La deuxième zone bénéficie d'un effet d'écran, les atteintes y sont donc moins prononcées: la défoliation est marquée mais incomplète, des anomalies de coloration sont visibles. La troisième zone, qui est la plus abritée vis-à-vis des embruns, conserve un aspect quasi normal avec peu ou pas de défoliation. Seuls les arbres de première ligne présentent ce type d'altération alors que les arbres situés plus à l'intèrieur ne sont pas affectés. Au fur et à mesure de la mort des rameaux ou des individus les plus proches du littoral, il y a progression du phénomène vers l'intérieur des terres. Très fréquemment, un effet d'écran très net est visible pour les arbres de seconde ligne. Les parties abritees des embruns restent parfaitement saines alors que les parties hautes ou latérales de ces arbres qui ne sont pas protégées présentent des symptômes marqués de dépérissement. En résumé, les dommages sont très étroitement liès à la position des individus ou des rameaux vis-à-vis de la mer. C'est cette localisation précise des atteintes qui a, très tôt, fait suspecter un rôle des embruns. Le fait que les dégâts affectent les arbres âgés de 150 à 200 ans, qui avaient jusqu'ici subi sans dommage l'action des aérosols marins, con-

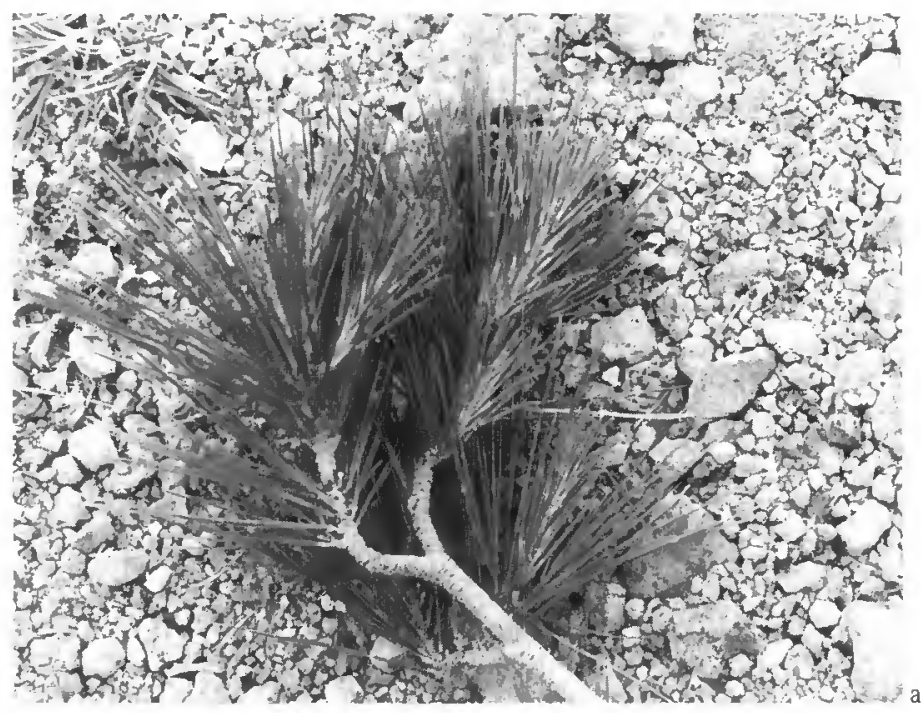
forte l'hypothèse de l'apparition " récente" dans les embruns d'un ou plusieurs composés phytotoxiques. Le phénomène coïncide avec l'augmentation massive des rejets en mer d'eaux usées, riches en détergents, et avec le développement du trafic maritime, qui est à l'origine de la libération d'hydrocarbures dans le milieu marin.

Des rameaux de Pin d'Alep soumis ou non à l'action d'embruns polluès sont présentès sur la photographie 2 (ci-dessus). La photographie 2a montre un rameau sain : la densité du feuillage est élevée et les aiguilles ont une coloration verte soutenue. Sur le rameau dépérissant de la photographie 2b, l'effet des embruns se fait d'abord sentir à l'extrèmité des aiguilles où dèbute la nécrose. La pointe de la feuille jaunit puis brunit. L'anomalie de coloration se propage ensuite vers le point d'insertion. Dans un stade ultérieur (photographie 2c), la totalité de l'aiguille se nécrose puis tombe. Le rameau prend alors rapidement un aspect beaucoup moins fourni. On constate également un dessèchement des bourgeons terminaux qui conduit à l'arrêt de crois- 


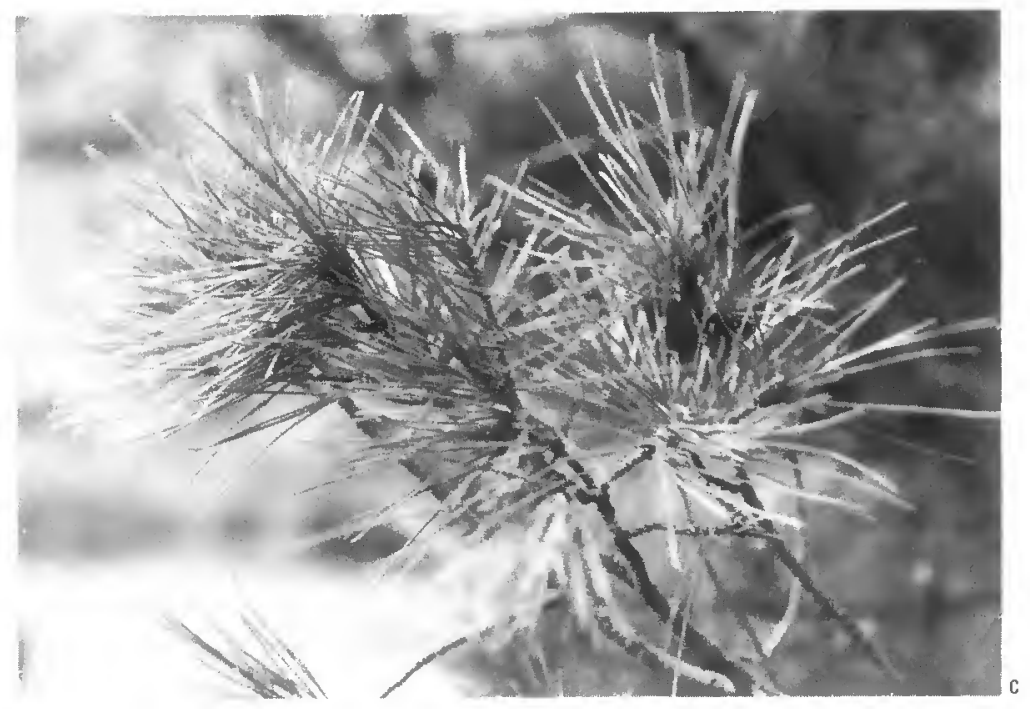

Photo 2

Rameaux de Pin d'Alep

présentant différents stades

de dépérissement.

a : rameau sain ;

b : rameau dépérissant;

c : rameau très dépérissant;

d: rameau sec.

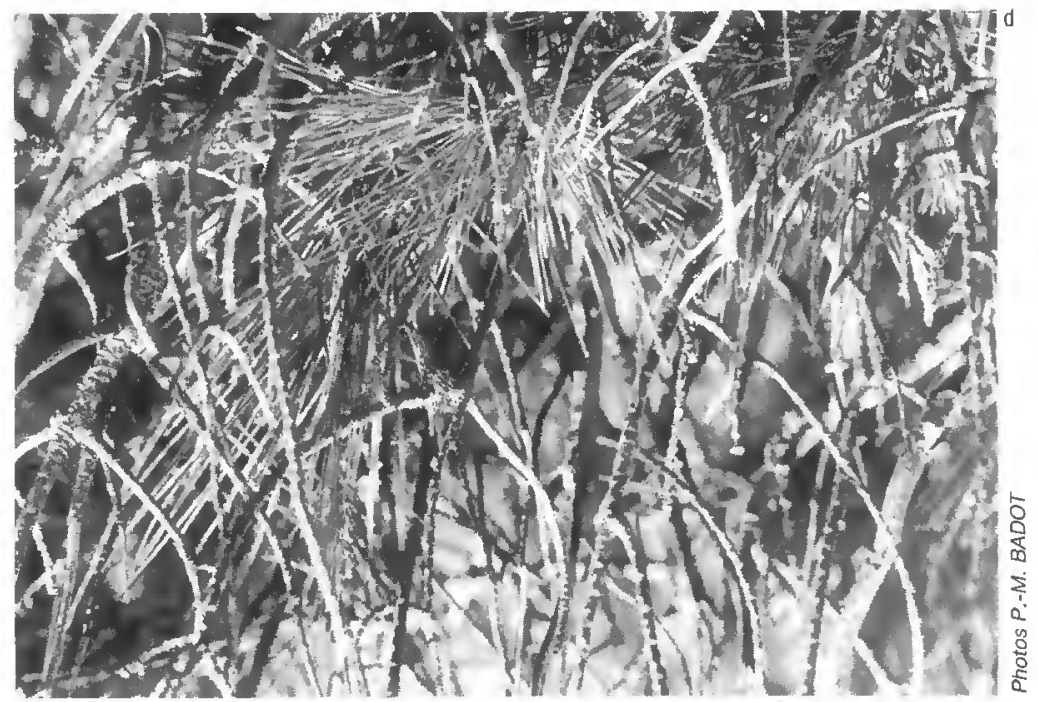

sance de la pousse. Dans quelques cas, nous avons également observé des anomalies morphologiques - aiguilles mal formées ou réduites à l'état d'écailles — indiquant que le fonctionnement méristématique est affecté. La défoliation a de graves conséquences à terme : un nombre réduit d'aiguilles limite la nutrition carbonée (diminution de la photosynthèse). On peut penser, de la même manière, que toutes les autres fonctions sont affectées. Effectivement, on a constaté, certaines années, l'arrêt complet de la croissance et du développement d'un grand nombre d'arbres implantés sur le littoral. Dans tous les cas, la croissance est réduite sous l'effet des embruns et très souvent la survie de l'arbre est compromise. Au stade ultime de dépérissement, toutes les aiguilles sont tombées et le rameau est alors entièrement sec (photographie $2 \mathrm{~d}$ ). II est important de noter que ces symptômes ne sont pas en relation avec d'éventuelles attaques parasitaires. 


\section{LOCALISATION GÉOGRAPHIQUE DES ZONES DÉPÉRISSANTES}

Des atteintes sont actuellement visibles en de très nombreux points du littoral méditerranéen. Les indications fournies sur la carte 1, ci-dessous, ont trait aux principales zones où des dépérissements ont été enregistrés. II s'agit ici de la synthèse des données disponibles dans la littérature et de nos propres observations: cette carte n'a qu'une valeur indicative et ne prétend pas à l'exhaustivité.

Le dépérissement est loin d'ētre uniforme le long de la Méditerranée. Deux facteurs principaux semblent être responsables de la répartition géographique des zones dépérissantes : le caractère plus ou moins abrupt et découpé de la côte et la proximité de grandes agglomérations ou de centres industrialo-portuaires importants. Les zones où la côte est tourmentée et accidentée sont moins atteintes que les zones de côtes basses, largement ouvertes aux vents de mer. Ainsi, les départements des Pyrénées-Orientales, de l'Aude et de l'Hérault sont plus sévèrement affectés que celui du Var qui est peu touché. Toutefois, dans ce dernier département, des atteintes significatives ont été rapportées dans la rade et les îles d'Hyères (Porquerolles et PortCros). Le flanc sud-est du massif du Dramont (commune de Saint-Raphaël) est également assez fortement affecté. Dans la région marseillaise, les dommages sont surtout visibles dans la région sud/sud-ouest. Dans les Alpes-Maritimes, des dégâts sont observés sur les îles de Lérins au large de Cannes, mais aussi de Roquebrune à Menton (R. Bigel, communication personnelle), ainsi qu'à Villeneuve-Loubet (parc de Vaugrenier). L'analyse de la répartition géographique du

\section{Carte 1 LOCALISATION DES ZONES DÉPÉRISSANTES}

Les flèches indiquent les zones oủ le dépérissement est assez général.

Les carrés correspondent à des sites précis où des atteintes très nettes sont observées.

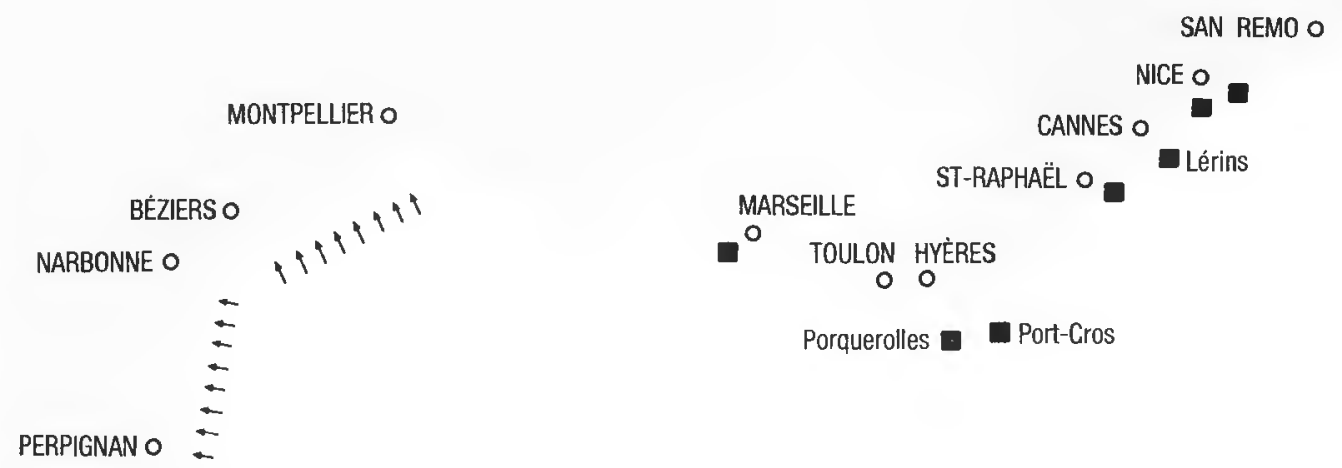


phènomène indique que la localisation des zones dépérissantes est liée de manière étroite à l'exposition aux vents marins. Ceci fournit ainsi un argument supplémentaire à l'appui de l'hypothèse selon laquelle ce sont les polluants présents dans les embruns qui sont responsables des dépérissements.

\section{LES MÉCANISMES POSSIBLES}

Depuis plusieurs années, des recherches ont èté effectuées par différentes équipes grâce au Parc national de Port-Cros, puis dans le cadre du groupement d'intérêt scientifique (GIS) Posidonie. Les travaux de l'équipe de J.-C. Sigoillot à Marseille, entre autres, ont permis de préciser certains points relatifs au mode d'action des embruns. II apparaît clairement qu'il est possible de reproduire les symptômes observés sur le terrain en soumettant la végétation à des mélanges de sels, d'hydrocarbures de type fuel et (ou) à des détergents comme les alkylbenzènes-sulfonates. Ces différentes substances seraient de plus capables d'agir en synergie. Par contre, lorsqu'ils sont appliqués seuls, les sels marins sont incapables de causer l'apparition de symptômes identiques à ceux enregistrés sur le terrain (Devèze et Sigoillot, 1978).

Les conditions météorologiques jouent un rôle très important dans la genèse des aérosols pollués. Lorsque les précipitations sont nulles ou peu abondantes et lorsque le vent est faible, les polluants s'accumulent en mer à l'interface air-eau dans les zones prèsentant une surface unie ou slick, qui apparaissent alors en bordure des côtes. Quand survient un épisode agitè, cette microcouche est entraînée par le vent selon des processus encore mal compris. Pendant le passage dans l'atmosphère, il se produit un nouvel enrichissement en polluants: les concentrations en détergents et en hydrocarbures dans les embruns peuvent être cent fois plus élevées que celles de l'eau de mer (Garrec et Sigoillot, 1992). Ce processus a pour origine le fait que les microgouttes qui constituent les embruns sont souvent entièrement évaporées, ce qui conduit à la formation d'un aérosol. Selon Devèze et Sigoillot (1978), les quantités de polluants transportées de cette manière sont très importantes. La végétation pourrait recevoir journellement jusqu'à $0,1 \mathrm{mg}$ de détergents anioniques, $1,5 \mathrm{mg}$ de produits pétroliers et $20 \mathrm{mg}$ de $\mathrm{NaCl}$ par mètre carré de surface fcliaire. De telles quantités sont du même ordre de grandeur que celles qui sont efficaces dans les expériences visant à reproduire en conditions contrôlées les symptômes observés sur le terrain.

Pour élucider les mécanismes exacts de la phytotoxicité des polluants sur les surfaces foliaires, un programme de recherche qui associe le laboratoire d'étude de la pollution atmosphérique de I'INRA de Nancy, le laboratoire de microbiologie de I'Université d'Aix-Marseille III et le laboratoire de botanique de l'Universitè de Franche-Comté vient d'être lancé avec le soutien du ministère de la Recherche et de l'Espace. Notre hypothèse de travail est la suivante: les substances impliquées - hydrocarbures et détergents - dégraderaient la couche lipidique présente au niveau de la cuticule qui recouvre la surface des feuilles. Les sels marins, mais aussi les différentes substances phytotoxiques, pourraient alors pénétrer dans les tissus foliaires où ils seraient alors à même d'exercer leurs effets néfastes. Nous cherchons parallèlement à mettre en évidence d'éventuelles perturbations hydriques dans les aiguilles des arbres atteints en relation avec les dégradations au niveau de la cuticule. Les premiers résultats obtenus montrent que les teneurs relatives en eau sont significativement réduites dans les arbres ou les rameaux dépérissants par rapport à ce qu'elles sont chez les arbres ou rameaux sains. Ceci tend à prouver le bien-fondé de notre hypothèse. Les premières observations, en microscopie èlectronique, des surfaces foliaires des aiguilles soumises à l'action des embruns montrent que la cuticule est altérée. Ces aiguilles seraient donc moins aptes à limiter leurs pertes d'eau, ce que confirme le fait qu'elles présentent des teneurs relatives en eau réduites. 
Les moyens d'action pour combattre ces dépérissements sont aujourd'hui limitès. II ne semble pas réaliste d'espérer à court terme une réduction suffisante de la pollution marine. Mieux comprendre le mode d'action des substances impliquées et leurs effets sur la croissance et le développement des arbres devrait cependant permettre de cerner la part respective prise par les différents polluants et de définir à terme les stratėgies de réduction de la pollution les plus appropriées.
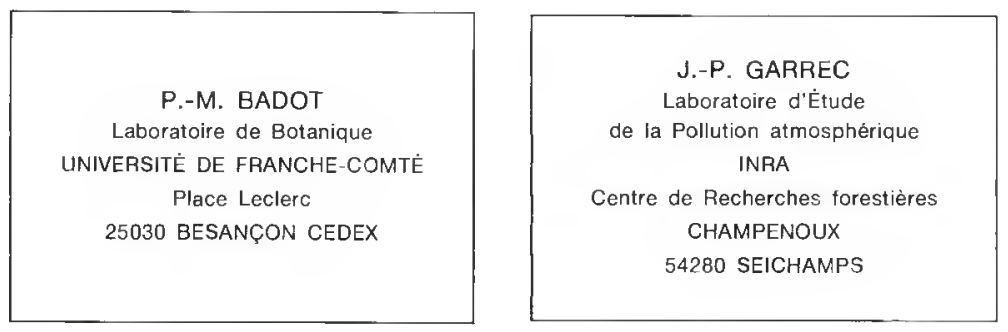

\section{Remerciements}

Ce travail bénéficie du soutien financier du ministère de la Recherche et de l'Espace. Les auteurs remercient V. Kulesa (ONF Nice) et G. Toussaint (ONF Saint-Raphaël) pour leurs précieuses indications concernant les zones dépérissantes.

\section{BIBLIOGRAPHIE}

BECKER (M.). - Incidence des conditions climatiques, édaphiques et sylvicoles sur la croissance et l'état de santé des forêts. - In : Les recherches en France sur le dépérissement des forêts. Programme DEFORPA, $2^{\circ}$ rapport / G. Landmann éd. - Nancy: École nationale du Génie rural, des Eaux et des Forēts, 1991, pp. 25-41.

BONNEAU (M.), LANDMANN (G.). - Le Dépérissement des forêts en Europe. - La Recherche, $n^{\circ} 205,1988$, pp. 1542-1553.

DEVĖZE (L.), SIGOILLOT (J.-C.). - Les Arbres malades de la mer. - Eau et Aménagement de la région provençale, $\mathrm{n}^{\circ} 19,1978$, pp. 13-24.

DOWDEN (H.G.M.), LAMBERT (M.J.). - Environmental factors associated with a disorder affecting tree species on the coast of New South Wales with particular reference to norfolk island pines Araucaria heterophylla. - Environmental Pollution, vol. 19, 1979, pp. 71-84.

GARREC (J.-P.). - Les jeunes Épicéas victimes de l'ozone. - La Recherche, $\mathrm{n}^{\circ} 236,1991, \mathrm{pp} .1228-1230$.

GARREC (J.-P.), SIGOILLOT (J.-C.). - Les Arbres malades de la mer. - La Recherche, vol. 23, $\mathrm{n}^{\circ} 245,1992$, pp. $940-941$.

GELLINI (R.), PANTANI (F.), GROSSONI (P.), BUSSOTI (F.), BARBOLANI (E.), RINALLO (C.). - Survey of the deterioration of the coastal vegetation in the park of San Rossore in Central Italy. - European Journal of Forest Pathology, vol. 13, 1983, pp. 296-304.

GIS-POSIDONIE. - Programme Embruns marins pollués. Rapport final. - Contrat SRETIE/MERE/89017, 1990, pp. 1-80.

LAPUCCI (P.I.), GELLINI (R.), PAIERO (P.). - Contaminazione chimica dell'acqua marina qualo causa di moria dei pini lungo le coste tirreniche.- Annali Accademia italiana di Scienze forestali, vol. 21, 1972, pp. 323358.

SIGOILLOT (J.-C.), NGUYEN (M.H.), DEVĖZE (L.). - Pollution par les aérosols marins dans les îles d'Hyères. - Travaux scientifiques du Parc national de Port-Cros, $\mathrm{n}^{\circ} 7,1981$, pp. 45-54. 